\title{
Composite Finite-time Convergent Guidance Law for Maneuvering Targets with Second-order Autopilot Lag
}

\author{
Junxiong $\mathrm{Wu}^{1,2}$, Hui Wang ${ }^{1,2 *}$, Shaoming $\mathrm{He}^{3}$ and Defu $\operatorname{Lin}^{1,2}$ \\ ${ }^{1}$ Beijing key laboratory of UAV Autonomous Control, Beijing Institute of Technology, Beijing, \\ 100081, People's Republic of China \\ ${ }^{2}$ School of Aerospace Engineering, Beijing Institute of Technology, Beijing, 100081, People's \\ Republic of China \\ ${ }^{3}$ School of Aerospace, Transport and Manufacturing, Cranfield University, Cranfield, UK.
}

\begin{abstract}
This paper aims to develop a new finite-time convergent guidance law for intercepting maneuvering targets accounting for second-order autopilot lag. The guidance law is applied to guarantee that the line of sight (LOS) angular rate converges to zero in finite time and results in a direct interception. The effect of autopilot dynamics can be compensated based on finite-time backstepping control method. The time derivative of the virtual input is avoided, taking advantage of integral-type Lyapunov functions. A finite-time disturbance observer (FTDOB) is used to estimate the lumped uncertainties and its high-order derivatives for improving the robustness and accuracy of the guidance system. Finite-time stability for the closed-loop guidance system is analyzed using Lyapunov function. Simulation results and comparisons are presented to illustrate the effectiveness of the guidance strategy.
\end{abstract}

Keywords: Finite-time backstepping control, Finite-time disturbance observer, Secondorder autopilot lag, Guidance law, Maneuvering targets

\section{Introduction}

Guidance law for tactical missiles plays an important role in the performance of intercepting maneuvering targets. The well-known proportional navigation (PN) guidance law has been proven to be effective in terms of simplicity, performance and ease of implementation [1-3]. Although the classical PN guidance law offers satisfactory performance for non-maneuvering or weakly maneuvering targets, it may not hold true in engaging highly maneuvering and agile targets, where the performance of PN guidance law drastically degrades [34]. To this end, advanced variants of the PN guidance law, such as augmented PN guidance law [1] are superior to the PN law. Guidance laws based on advanced nonlinear control theories and robust control theories can also be found, such as H-infinity guidance law [4], Lyapunov-based nonlinear guidance law [5], $L_{2}$ gain guidance law [6], differential game guidance laws [7, 8],

\footnotetext{
*Corresponding author:

Hui Wang, School of Aerospace Engineering, Beijing Institute of Technology, Beijing, 100081, People's Republic of China.
}

Email: wh20031131@126.com 
and sliding mode control based guidance laws [9-12].

These laws generally require explicit information of target acceleration. Nonetheless, the knowledge of the target motion is not usually available from sensors on board. Thus, implementation of such guidance laws becomes restrictive. In this regard, target acceleration has been frequently regarded as external disturbance and compensated as feedforward term. Disturbance observer (DOB) is a well-known approach used to estimate disturbance of system and improve the performance of the controller [13-15]. In [15], a guidance law is designed based on integral sliding mode control method, and a nonlinear DOB is used to eliminate the influence of target maneuvers. A formulation of terminal guidance law for missile intercepting maneuvering target is investigated in [16]; in this study, a nonlinear DOB is employed to improve the system performance and avoid the chattering phenomenon.

In many realistic scenarios such as defense against incoming high-speed tactical ballistic missiles (TBMs), the interception time of the end-game scenario is only several seconds. Therefore, the LOS angular rate is required to converge rapidly to zero to maintain the collision triangle. Finite-time convergent (FTC) control law is an effective feedback control methodology that exhibits high robustness and convergence rate against uncertainty. The authors in [17] propose a novel finite-time convergent guidance law to prove that LOS angular rate converges to zero or the presence of a small range around zero before interception. A nonsingular terminal sliding mode based guidance law is developed in [18] to guarantee finite-time stability for maneuvering target interception. However, the undesired chattering phenomenon is the main limitation for implementing these guidance laws.

An autopilot lag exists between command and acceleration missile achieved; this lag exerts an undesirable effect on the performance of the guidance system. The integrated design of missile autopilot and guidance system may improve guidance precision. In $[19,20]$, guidance laws considering first-order autopilot dynamics are designed based on backstepping method. However, missile autopilot is always considered a complex system, and the properties of autopilot can be well approximated by the second-order lag rather than the first-order lag [21-26]. The authors in [25] present a guidance law with second-order autopilot dynamics compensation, but only asymptotical stability is proven. Accounting for second-order autopilot lag, a finite-time convergent guidance law based on standard backstepping control technique is developed in [24]. The required analytic differentiations of intermediate controls are obtained using a tracking differentiator.

Motivated the above discussion, this work investigates a new composite finite-time convergent guidance law in the presence of second-order autopilot dynamics. The new guidance law is a disturbance observer based controller that can faster rejection of disturbances and recovery of nominal performances than pure feedback control. Two studies [29] and [32] inspired our work. Authors in [29] developed a finite-time backstepping method and finite-time feedback controllers for a class of nonlinear 
systems. This control method is used here to compensate autopilot lag. Based on finitetime controller, our paper introduces a noise insensitivity FTDOB [32] to estimate the unknown target acceleration for improving guidance performance. Another attractive property compared with traditional backstepping procedure is that the new guidance law does not require the analytic derivative of virtual control law in the derivation process. A two-step stability analysis of the closed-loop system is established conducted using finite-time bounded (FTB) function and Lypunov function. Finally, simulations are performed considering various cases of highly maneuvering targets for varying missile velocity profiles to demonstrate the effectiveness of the proposed guidance law.

This paper is organized as follows. Section 2 presents some basic concepts and formulations of nonlinear model of engagement. Section 3 focuses on the detailed design procedure of the proposed guidance law. Section 4 presents the results of closedloop system stability. Section 5 states the simulation results and comparison of the proposed law with existing formulations. Finally, Section 6 discusses the conclusions.

\section{Preliminaries and problem formation}

\subsection{Some lemmas}

The following lemmas should be recalled because they play an important role in subsequent study of finite-time convergent guidance law.

Lemma 1: [28] Assume a positive definite, continuously differentiable function $V(x)$ defined on $U \in R^{n}$ and real numbers $c>0$ and $0<\alpha<1$, such that $\dot{V}(x)+c(V(x))^{\alpha}$ is a negative semi-definite function on $U \in R^{n}$; an area $U_{0} \subset R^{n}$ that exists and any $V(x)$ starts from $U_{0} \subset R^{n}$ can reach $V(x) \equiv 0$ in finite time

$$
T_{\text {reach }} \leq \frac{\left(V\left(x_{0}\right)\right)^{1-\alpha}}{c(1-\alpha)}
$$

where $V\left(x_{0}\right)$ is the initial value of $V(x)$.

Lemma 2: [29] If $p_{1}>0$ and $0<p_{2} \leq 1$, then $\forall x \in R, \forall y \in R$

$$
\left|x^{p_{1} p_{2}}-y^{p_{1} p_{2}}\right| \leq 2^{1-p_{2}}\left|x^{p_{1}}-y^{p_{1}}\right|^{p_{2}}
$$

Lemma 3: [29] Let $c$ and $d$ be positive constants. Given any positive number $\gamma>0$, the following inequality holds:

$$
|x|^{c}|y|^{d} \leq \frac{c}{c+d} \gamma|x|^{c+d}+\frac{d}{c+d} \gamma^{-c / d}|y|^{c+d}
$$

Lemma 4: [29] For any real numbers $x_{i}, i=1, \ldots, n$ and $0<b \leq 1$, the following inequality holds:

$$
\left(\left|x_{1}\right|+\cdots+\left|x_{n}\right|\right)^{b} \leq\left|x_{1}\right|^{b}+\cdots+\left|x_{n}\right|^{b}
$$

2.2 Nonlinear engagement of pursuit-evasion motion 


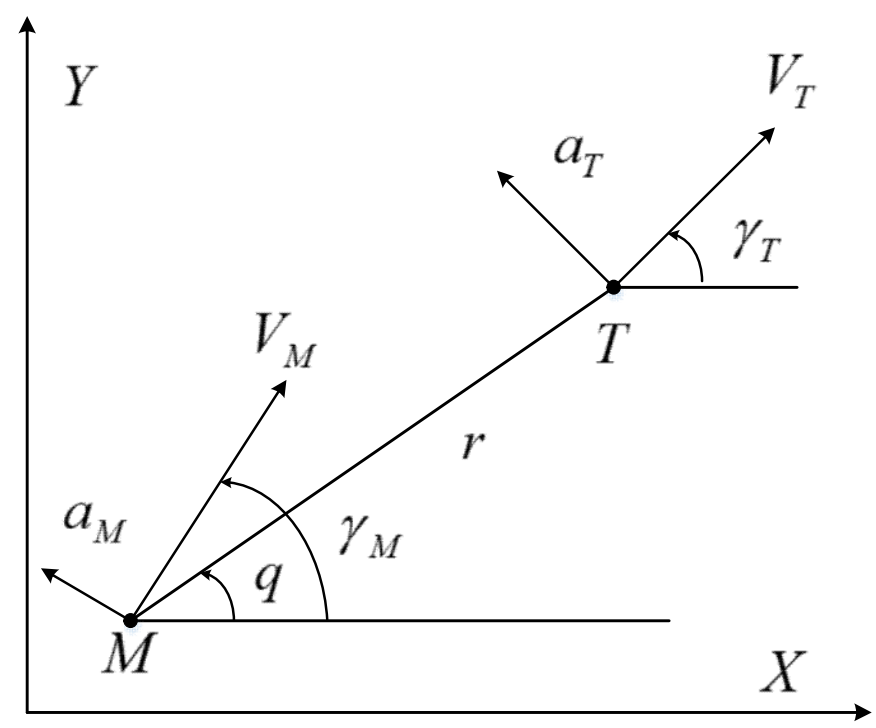

Fig. 1. 2D target-missile engagement geometry

The planar engagement scenario which consists of a missile pursuing a maneuvering target is shown in Fig. 1, where symbols $M$ and $T$ denote a missile and a target, respectively; the flight path angles of the missile and target are represented by $\gamma_{M}$ and $\gamma_{T}$, respectively; $r$ is the relative range between the target and missile; $q$ is the LOS angle; the velocities of the missile and target are represented by $V_{M}$ and $V_{T}$, respectively; and the accelerations of the missile and target are represented by $a_{M}$ and $a_{T}$, respectively.

In practical, end-game scenario has no thrust and aerodynamics serves as control input. We assume that the command acceleration of the missile is perpendicular to its velocity vector. The missile and the target are assumed to be point masses to simplify dynamics of engagement. Furthermore, the magnitudes of velocity of the missile and target are assumed constant.

Under the above-stated assumptions, the corresponding equations of motion that depict the engagement scenario are formulated as [27]

$$
\begin{gathered}
\dot{r}=V_{T} \cos \left(\gamma_{T}-q\right)-V_{M} \cos \left(\gamma_{M}-q\right) \\
\dot{q}=\frac{1}{r}\left[V_{T} \sin \left(\gamma_{T}-q\right)-V_{M} \sin \left(\gamma_{M}-q\right)\right] \\
\dot{\gamma}_{M}=\frac{a_{M}}{V_{M}} \\
\dot{\gamma}_{T}=\frac{a_{T}}{V_{T}}
\end{gathered}
$$

Let $V_{r}=\dot{r}, V_{q}=r \dot{q}$, then, differentiating Eqs. (1)-(2) and rearranging the terms yield [31]: 


$$
\begin{gathered}
\dot{V}_{r}=\frac{V_{q}^{2}}{r}+a_{T r}-a_{M r} \\
\dot{V}_{q}=-\frac{V_{r} V_{q}}{r}+a_{T q}-a_{M q}
\end{gathered}
$$

where, $\quad a_{T r}=a_{T} \sin \left(q-\gamma_{T}\right), \quad a_{M r}=a_{M} \sin \left(q-\gamma_{M}\right), \quad a_{T q}=a_{T} \cos \left(q-\gamma_{T}\right), \quad$ and $a_{M q}=a_{M} \cos \left(q-\gamma_{M}\right) . a_{M r}$ and $a_{T r}$ represent the projections of the missile and the target acceleration along the LOS, respectively. $a_{M q}$ and $a_{T q}$ are the projections of the missile and the target acceleration orthogonal to LOS, respectively. The target acceleration $a_{T}$ is usually difficult to measure directly [31]. Therefore $a_{T r}$ and $a_{T q}$ are considered as unknown disturbances.

Normally, the acceleration along missile velocity of most tactical missiles in terminal phase cannot be controlled, and a well-known guidance strategy is nullify the LOS angular rate $\dot{q}$ [17]. Thus, the purpose of guidance system is adjusting lateral acceleration $a_{M}$ to keep $\dot{q}$ in a small range around zero and end up with a successful interception. Moreover, to remove the combined effect of nonlinear term and unknown target maneuver, a new quantity is defined as follows:

$$
h=-\frac{V_{r} V_{q}}{r}+a_{T q}-a_{M} \cos \left(q-\gamma_{M}\right)+a_{M}
$$

then Eq. (6) can be rewritten as

$$
\dot{V}_{q}=h-a_{M}
$$

Assumption 1: Consider a positive constant $\sigma$ and a positive integer $m$, the lumped uncertainty $h$ is piecewise continuous and satisfies

$$
\left|\frac{d^{m} h}{d t^{m}}\right| \leq \sigma
$$

Remark 1: During the guided flight process, both the missile and target have physical limitations, namely, coefficients which compose lumped uncertainty are time-varying bounded. Thus, the assumption considered above is not restrictive.

In practice, the missile autopilot can be well approximated by the second-order system as [25]:

$$
\ddot{a}_{M}=-2 \xi_{a} \omega_{a} \dot{a}_{M}-\omega_{a}^{2} a_{M}+\omega_{a}^{2} a_{M c}
$$

where $a_{M}$ denotes the achieved missile acceleration, $a_{M c}$ is the commanded acceleration of missile, $\xi_{a}$ and $\omega_{a}$ denote the damping ration and natural frequency, respectively, of the missile autopilot. However, the performance of missile is adversely affected by the autopilot lag between the commanded acceleration and real acceleration of missile. Compensation of dynamics is an efficacious way to remove the influence of system lag. 
The objective of this paper is to design a guidance law in the presence of unknown target maneuver and autopilot dynamics. Based on derived guidance law, the LOS angular rate of the close-loop system is regulated to zero in finite time.

\section{Guidance law design with finite-time convergence}

A finite-time convergent guidance law is presented by using finite-time backstepping control technique and FTDOB in this section. The systematic procedure of guidance law design and proof of close-loop finite-stability are given by the following.

\subsection{Control algorithm}

We define state variables $x_{1}=V_{q}, x_{2}=-a_{M}$, and $x_{3}=-\dot{a}_{M}$. An integrated guidance and control system can be written as follows:

$$
\begin{aligned}
& \dot{x}_{1}=h+x_{2} \\
& \dot{x}_{2}=x_{3} \\
& \dot{x}_{3}=-2 \xi_{a} \omega_{a} x_{3}-\omega_{a}^{2} x_{2}-\omega_{a}^{2} a_{M c}
\end{aligned}
$$

An FTDOB [32] is employed here to estimate disturbance and compensate control input to suppress the lumped uncertainty $h$. A third-order observer can then be constructed as follows:

$$
\begin{aligned}
& \dot{z}_{0}=v_{0}+x_{2}, v_{0}=-\lambda_{3} L^{1 / 4}\left|z_{0}-x_{1}\right|^{3 / 4} \operatorname{sgn}\left(z_{0}-x_{1}\right)+z_{1} \\
& \dot{z}_{1}=v_{1}, v_{1}=-\lambda_{2} L^{1 / 3}\left|z_{1}-v_{0}\right|^{2 / 3} \operatorname{sgn}\left(z_{1}-v_{0}\right)+z_{2} \\
& \dot{z}_{2}=v_{2}, v_{2}=-\lambda_{1} L^{1 / 2}\left|z_{2}-v_{1}\right|^{1 / 2} \operatorname{sgn}\left(z_{2}-v_{1}\right)+z_{3} \\
& \dot{z}_{3}=-\lambda_{0} L \operatorname{sgn}\left(z_{3}-v_{2}\right)
\end{aligned}
$$

where $\lambda_{i}>0(i=0,1,2,3)$ and $L>0$ are the observer gains to be designed, with appropriate values of observer gains, $z_{0}, z_{1}, z_{2}, z_{3}$ approaches to $V_{q}, h, \dot{h}, \ddot{h}$, respectively. According to study of Levant [32], the following Lemma is obtained.

Lemma 5: Under Assumption 1, the estimation error dynamics of FTDOB (Eq. 12) is governed by:

$$
\begin{aligned}
& \dot{\sigma}_{0}=-\lambda_{3} L^{1 / 4}\left|\sigma_{0}\right|^{3 / 4} \operatorname{sgn}\left(\sigma_{0}\right)+\sigma_{1} \\
& \dot{\sigma}_{1}=-\lambda_{2} L^{1 / 3}\left|\sigma_{1}-\dot{\sigma}_{0}\right|^{3 / 4} \operatorname{sgn}\left(\sigma_{1}-\dot{\sigma}_{0}\right)+\sigma_{2} \\
& \dot{\sigma}_{2}=-\lambda_{1} L^{1 / 2}\left|\sigma_{2}-\dot{\sigma}_{1}\right|^{1 / 2} \operatorname{sgn}\left(\sigma_{2}-\dot{\sigma}_{1}\right)+\sigma_{3} \\
& \dot{\sigma}_{3} \in-\lambda_{0} L \operatorname{sgn}\left(z_{n}-v_{n-1}\right)+[-L, L]
\end{aligned}
$$

where the inclusion $\ddot{h} \in[-L, L]$ is used in the last line, $\sigma_{0}=z_{0}-x_{1}, \sigma_{1}=z_{1}-h$, $\sigma_{2}=z_{2}-\dot{h}$, and $\sigma_{3}=z_{3}-\ddot{h}$ are the estimation errors. A time constant exists $t_{s}>0$ such that $\sigma_{i}=0 \quad(i=0,1,2,3)$ for all $t>t_{s}$ because the observer is finite-time stable. Remark 2: The principle selection of observer gains $\lambda_{i}>0(i=0,1,2,3)$ and $L>0$ can be followed from [32]. The convergent rate will be very fast when the observer gain $L$ is large enough, but a very large value of $L$ will lead to unexpected overshoot and an excessive acceleration command. Hence, $L$ should be designed under certain 
circumstance.

Remark 3: The defect of lumped uncertainty, concludes from error dynamics (13) that $\sigma_{0}=\sigma_{1}=\sigma_{2}=\sigma_{3}=0$ always holds when the initial states are set as $z_{0}(0)=V_{q}$, $z_{1}(0)=0, z_{2}(0)=0$, and $z_{3}(0)=0$. Hence, the FTDOB has no influence on closedloop system and the performance of nominal system is retained. In addition, the uncertainty can be estimated regardless of control input, then the FTDOB and guidance law can be designed independently.

The integrated system can be expressed as follows

$$
\begin{aligned}
& \dot{x}_{1}=z_{1}-\left(z_{1}-h\right)+x_{2} \\
& \dot{x}_{2}=x_{3} \\
& \dot{x}_{3}=-2 \xi_{a} \omega_{a} x_{3}-\omega_{a}^{2} x_{2}-\omega_{a}^{2} a_{M c}
\end{aligned}
$$

$z_{1}$ will converge to uncertainty $h$ when $t>t_{s}$. According to Lemma 5, system reduces to:

$$
\begin{aligned}
& \dot{x}_{1}=x_{2}+z_{1} \\
& \dot{x}_{2}=x_{3} \\
& \dot{x}_{3}=-2 \xi_{a} \omega_{a} x_{3}-\omega_{a}^{2} x_{2}-\omega_{a}^{2} a_{M c}
\end{aligned}
$$

The nonlinear system (15) is in a parametric strict feedback form. The backstepping method is an effective way to cancel influence of uncertainty for aforementioned system. Similar to traditional backstepping method, a finite-time backstepping control method proposed in [29] is used to deduce guidance law step by step as follows.

Step 1: Let $\bar{x}_{1}=x_{1}, \bar{x}_{2}=x_{2}+z_{1}, \bar{x}_{3}=x_{3}+\dot{z}_{1}$, system (15) can be rewritten as:

$$
\begin{aligned}
& \dot{\bar{x}}_{1}=\bar{x}_{2} \\
& \dot{\bar{x}}_{2}=\dot{\bar{x}}_{3} \\
& \dot{\bar{x}}_{3}=-2 \xi_{a} \omega_{a}\left(\bar{x}_{3}-\dot{z}_{1}\right)-\omega_{a}^{2}\left(\bar{x}_{2}-z_{1}\right)-\omega_{a}^{2} a_{M c}+\ddot{z}_{1}
\end{aligned}
$$

Let $\tau=p / q \in\left(\begin{array}{ll}0 & 1 / 3\end{array}\right)$ and $q_{k}=1-(k-1) \tau$, where $p$ is an even integer and $q$ is an odd integer. We have $1=q_{1}>q_{2}>\cdots>q_{n+1}>0, \quad q_{i+1}=q_{2}+q_{i}-1$, and $q_{i}-q_{i+1}=\tau$. A $C^{1}$, positive definite and proper Lyapunov function is chosen as follows: [33]

$$
V_{k}\left(\bar{x}_{1}, \ldots, \bar{x}_{k}\right)=\sum_{l=1}^{k} W_{l}, W_{l}=\int_{\bar{x}_{l}^{*}}^{\bar{x}_{l}}\left(s^{1 / q_{l}}-\left(\bar{x}_{l}^{*}\right)^{1 / q_{l}}\right)^{2-q_{l}} d s
$$

where $\bar{x}_{k}^{*}$ represents the virtual control law, defined by

$$
\begin{array}{ll}
\bar{x}_{1}^{*}=0 & \xi_{1}=\bar{x}_{1}^{1 / q_{1}}=\bar{x}_{1} \\
\vdots & \vdots \\
\bar{x}_{k}^{*}=-\kappa_{k-1} \xi_{k-1}^{q_{k}} & \xi_{k}=\bar{x}_{k}^{1 / q_{k}}-\left(\bar{x}_{k}^{*}\right)^{1 / q_{k}}
\end{array}
$$

with constants $\kappa_{k}>0$. 
Taking the derivative of $V_{1}\left(\bar{x}_{1}\right)$ along system (16) produces:

$$
\dot{V}_{1}\left(\bar{x}_{1}\right)=\left(\bar{x}_{1}\right)^{2-q_{1}}\left(\bar{x}_{2}^{*}+\bar{x}_{2}-\bar{x}_{2}^{*}\right)=\xi_{1}\left(\bar{x}_{2}-\bar{x}_{2}^{*}\right)-\kappa_{1}\left(\xi_{1}\right)^{2-\tau}
$$

Step 2: In the following proposition, we can obtain a useful property proven in [33].

Proposition 1: $W_{k}\left(\bar{x}_{1}, \ldots, \bar{x}_{k}\right)$ is $C^{1}, \partial W_{k} / \partial \bar{x}_{k}=\xi_{k}^{2-q_{k}}$ and for $l=1, \ldots, k-1$

$$
\frac{\partial W_{k}}{\partial \bar{x}_{l}}=-\left(2-q_{k}\right) \frac{\partial\left(\bar{x}_{k}^{*}\right)^{1 / q_{k}}}{\partial \bar{x}_{l}} \int_{\bar{x}_{k}^{*}}^{\bar{x}_{k}}\left(s^{1 / q_{k}}-\left(\bar{x}_{k}^{*}\right)^{1 / q_{k}}\right)^{2-q_{k}} d s
$$

According to Proposition 1, computing the first-time derivative of $V_{2}$ gives:

$$
\begin{aligned}
& \dot{V}_{2}\left(\bar{x}_{1}, \bar{x}_{2}\right)=\dot{V}_{1}+\frac{\partial W_{2}}{\partial \bar{x}_{1}} \dot{\bar{x}}_{1}+\frac{\partial W_{2}}{\partial \bar{x}_{2}} \dot{\bar{x}}_{2} \\
& =-\kappa_{1} \xi_{1}^{2-\tau}+\xi_{1}\left(\bar{x}_{2}-\bar{x}_{2}^{*}\right)+\frac{\partial W_{2}}{\partial \bar{x}_{1}} \dot{\bar{x}}_{1}+\xi_{2}^{2-q_{2}} \dot{\bar{x}}_{2}
\end{aligned}
$$

From Lemma 2 and Lemma3, the equation is

$$
\xi_{1}\left(\bar{x}_{2}-\bar{x}_{2}^{*}\right) \leq 2\left|\xi_{1}\right|\left|\xi_{2}\right|^{q_{2}} \leq \frac{2}{2-\tau}\left|\xi_{1}\right|^{2-\tau}+\frac{2 q_{2}}{2-\tau}\left|\xi_{2}\right|^{2-\tau} \leq c_{21}\left|\xi_{1}\right|^{2-\tau}+c_{22}\left|\xi_{2}\right|^{2-\tau}
$$

where $c_{21}>2 /(2-\tau)$ and $c_{22}>2 q_{2} /(2-\tau)$ are positive constants.

We introduce following proposition whose proof are given in the appendix to facilitate the construction of finite-time controller and estimate the right-hand side of Eq. (20).

Proposition 2: $\bar{\rho}_{21}$ and $\bar{\rho}_{22}$ are positive constants such that:

$$
\frac{\partial W_{2}}{\partial \bar{x}_{1}} \dot{\bar{x}}_{1} \leq \bar{\rho}_{21}\left|\xi_{1}\right|^{2-\tau}+\bar{\rho}_{22}\left|\xi_{2}\right|^{2-\tau}
$$

Substituting (21) and (22) into (20) gives:

$$
\begin{aligned}
\dot{V}_{2}\left(x_{1}, x_{2}\right) \leq\left(-\kappa_{1}+c_{21}\right. & \left.+\bar{\rho}_{21}\right)\left|\xi_{1}\right|^{2-\tau}+\left(c_{22}+\bar{\rho}_{22}\right)\left|\xi_{2}\right|^{2-\tau} \\
& -\kappa_{2}\left|\xi_{2}\right|^{2-\tau}+\xi_{2}^{2-q_{2}}\left(\bar{x}_{3}-\bar{x}_{3}^{*}\right)
\end{aligned}
$$

Step 3: According to Proposition 1, evaluating the first-time derivative of $V_{3}$ gives:

$$
\begin{aligned}
& \dot{V}_{3}\left(\bar{x}_{1}, \bar{x}_{2}, \bar{x}_{3}\right)=\dot{V}_{2}+\frac{\partial W_{3}}{\partial \bar{x}_{1}} \dot{\bar{x}}_{1}+\frac{\partial W_{3}}{\partial \bar{x}_{2}} \dot{\bar{x}}_{2}+\frac{\partial W_{3}}{\partial \bar{x}_{3}} \dot{\bar{x}}_{3} \\
& \leq\left(-\kappa_{1}+c_{21}+\bar{\rho}_{21}\right)\left|\xi_{1}\right|^{2-\tau}+\left(c_{22}+\bar{\rho}_{22}\right)\left|\xi_{2}\right|^{2-\tau}-\kappa_{2}\left|\xi_{2}\right|^{2-\tau} \\
& +\xi_{2}^{2-q_{2}}\left(\bar{x}_{3}-\bar{x}_{3}^{*}\right)+\frac{\partial W_{3}}{\partial \bar{x}_{1}} \dot{\bar{x}}_{1}+\frac{\partial W_{3}}{\partial \bar{x}_{2}} \dot{\bar{x}}_{2}+\xi_{3}^{2-q_{3}} \dot{\bar{x}}_{3}
\end{aligned}
$$

Similar to derivation process in Step 2 we have:

$$
\xi_{2}^{2-q_{2}}\left(\bar{x}_{3}-\bar{x}_{3}^{*}\right) \leq c_{31}\left|\xi_{2}\right|^{2-\tau}+c_{32}\left|\xi_{3}\right|^{2-\tau}
$$

where $c_{31}>2\left(2-q_{2}\right) /(2-\tau)$ and $c_{32}>2 q_{3} /(2-\tau)$ are positive constants.

The proposition whose proof is given in the appendix follows.

Proposition 3: $\bar{\rho}_{31}, \bar{\rho}_{32}$ and $\bar{\rho}_{33}$ are positive constants such that 


$$
\frac{\partial W_{3}}{\partial \bar{x}_{1}} \dot{\bar{x}}_{1}+\frac{\partial W_{3}}{\partial \bar{x}_{2}} \dot{\bar{x}}_{2} \leq \bar{\rho}_{31}\left|\xi_{1}\right|^{2-\tau}+\bar{\rho}_{32}\left|\xi_{2}\right|^{2-\tau}+\bar{\rho}_{33}\left|\xi_{3}\right|^{2-\tau}
$$

Substituting (25) and (26) into (24) gives

$$
\begin{aligned}
& \dot{V}_{3} \leq\left(-\kappa_{1}+c_{21}+\bar{\rho}_{21}+\bar{\rho}_{31}\right)\left|\xi_{1}\right|^{2-\tau}+\left(-\kappa_{2}+c_{22}+c_{31}+\bar{\rho}_{22}+\bar{\rho}_{32}\right)\left|\xi_{2}\right|^{2-\tau} \\
& +\left(c_{32}+\bar{\rho}_{33}\right)\left|\xi_{3}\right|^{2-\tau}+\xi_{3}^{2-q_{3}}\left(-2 \xi_{a} \omega_{a}\left(\bar{x}_{3}-\dot{z}_{1}\right)-\omega_{a}^{2}\left(\bar{x}_{2}-z_{1}\right)-\omega_{a}^{2} a_{M c}+\ddot{z}_{1}\right)
\end{aligned}
$$

The proposed acceleration command is obtained as

$$
a_{M c}=-\frac{1}{\omega_{a}^{2}}\left(-\kappa_{3} \xi_{3}^{q_{4}}+2 \xi_{a} \omega_{a}\left(\bar{x}_{3}-\dot{z}_{1}\right)+\omega_{a}^{2}\left(\bar{x}_{2}-z_{1}\right)-\ddot{z}_{1}\right)
$$

Finally, according to Lemma $5, z_{1}, z_{2}$, and $z_{3}$ will converge to $h, \dot{h}$, and $\ddot{h}$ respectively, in finite time. Definitions of $\bar{x}_{2}, \bar{x}_{3}$, and the guidance law (28) can be replaced with:

$$
a_{M c}=-\frac{1}{\omega_{a}^{2}}\left(-\kappa_{3} \xi_{3}^{q_{4}}+2 \xi_{a} \omega_{a} x_{3}+\omega_{a}^{2} x_{2}-z_{3}\right)
$$

By using integral-type Lyapunov functions, the analytic differentiation of virtual control law can be completely avoided in a backstepping-like procedure to induce proposed guidance law.

3.2 Closed-loop stability analysis

Theorem 1: Consider integrated system (15), the proposed guidance law (29) with FDOB (12) will drive the LOS angular rate to zero in finite time.

\section{Proof:}

Nonlinear observer-controller structure is not usually suitable for the well-known separation principle. Therefore, the proof of Theorem 1 is divided into two main steps. First, the boundness of the system states is proven during the convergent phase of FTDOB based on FTB function technique. Next, the closed-loop finite-time stability of the overall system will be presented with Lyapunov function approach.

Step 1: The candidate FTB function for system (14) is defined as:

$$
V=\frac{1}{2} \sum_{i=1}^{3} x_{i}^{2}+\frac{1}{2} \sum_{i=0}^{3} z_{i}^{2}
$$

The derivative of $V$ along system trajectory (14) yields:

$$
\begin{aligned}
& \dot{V}=x_{1} \dot{x}_{1}+x_{2} \dot{x}_{2}+x_{3} \dot{x}_{3}+z_{0} \dot{z}_{0}+z_{1} \dot{z}_{1}+z_{2} \dot{z}_{2}+z_{3} \dot{z}_{3} \\
& =x_{1}\left(x_{2}+z_{1}-\sigma_{1}\right)+x_{2} x_{3}+x_{3}\left(-2 \xi_{a} \omega_{a} x_{3}-\omega_{a}^{2} x_{2}-\omega_{a}^{2} a_{M c}\right)+z_{0}\left(v_{0}+x_{2}\right) \\
& +z_{1} v_{1}+z_{2} v_{2}+z_{3}\left(-\lambda_{0} L \operatorname{sgn}\left(z_{3}-v_{2}\right)\right)
\end{aligned}
$$

With $\forall x \in R, 0<\tau<1$, the inequality $|x|^{\tau}<1+|x|$ holds. Lemma 4 can deduce that:

$$
\begin{aligned}
& \left|v_{0}\right|=\left|-\lambda_{3} L^{1 / 4}\right| z_{0}-\left.x_{1}\right|^{3 / 4} \operatorname{sgn}\left(z_{0}-x_{1}\right)+\left.z_{1}\left|\leq \lambda_{3} L^{1 / 4}\right| z_{0}\right|^{3 / 4}+\lambda_{3} L^{1 / 4}\left|x_{1}\right|^{3 / 4}+\left|z_{1}\right| \\
& \leq \lambda_{3} L^{1 / 4}\left(\left|z_{0}\right|+1\right)+\left|z_{1}\right|+\lambda_{3} L^{1 / 4}\left|x_{1}\right|^{3 / 4}
\end{aligned}
$$


Note that $\left|x_{i}\right| \leq \sqrt{2 V}, i=1,2,3,\left|z_{j}\right| \leq \sqrt{2 V}$, and $i=0,1,2,3$. Besides, during the terminal phase, the closing velocity along and perpendicular to the LOS, as well as the missile-target relative distance are bounded in any time interval $[0, t]$; hence, $V_{r}, V_{q}$, and $r$ all have limited values during any time interval $[0, t]$. The existence of positive constants $\delta_{v_{0}}, L_{v_{0}}$ satisfies the following:

$$
\left|v_{0}\right| \leq \delta_{v_{0}} \sqrt{V}+L_{v_{0}}
$$

Likewise, we conclude that

$$
\begin{aligned}
& \left|v_{1}\right|=\left|-\lambda_{2} L^{1 / 3}\right| z_{1}-\left.v_{0}\right|^{2 / 3} \operatorname{sgn}\left(z_{1}-v_{0}\right)+z_{2}\left|\leq \lambda_{2} L^{1 / 3}\right| z_{1}-\left.v_{0}\right|^{2 / 3}+\left|z_{2}\right| \\
& \leq \lambda_{2} L^{1 / 3}\left(\left|z_{1}\right|+2\right)+\left|z_{2}\right|+\lambda_{2} L^{1 / 3}\left|v_{0}\right| \leq \lambda_{2} L^{1 / 3}\left(\left|z_{1}\right|+2\right)+\left|z_{2}\right|+\lambda_{2} L^{1 / 3}\left(\delta_{v_{0}} \sqrt{V}+L_{v_{0}}\right) \\
& \leq \delta_{v_{1}} \sqrt{V}+L_{v_{1}} \\
& \left|v_{2}\right|=\left|-\lambda_{1} L^{1 / 2}\right| z_{2}-\left.v_{1}\right|^{1 / 2} \operatorname{sgn}\left(z_{2}-v_{1}\right)+z_{3}\left|\leq \lambda_{1} L^{1 / 2}\right| z_{2}-\left.v_{1}\right|^{1 / 2}+\left|z_{3}\right| \\
& \leq \lambda_{1} L^{1 / 2}\left(\left|z_{2}\right|+2\right)+\left|z_{3}\right|+\lambda_{1} L^{1 / 2}\left|v_{1}\right| \leq \lambda_{1} L^{1 / 2}\left(\left|z_{2}\right|+2\right)+\left|z_{3}\right|+\lambda_{1} L^{1 / 2}\left(\delta_{v_{1}} \sqrt{V}+L_{v_{1}}\right) \\
& \leq \delta_{v_{2}} \sqrt{V}+L_{v_{2}} \\
& \quad\left|\omega_{a}^{2} a_{M c}\right| \leq\left|-\kappa_{3} \xi_{3}^{q_{4}}+2 \xi_{a} \omega_{a} x_{3}+\omega_{a}^{2} x_{2}-z_{3}\right| \\
& \quad \leq \kappa_{3}\left(\left|x_{3}\right|^{q_{4} / q_{3}}+\left|z_{2}\right|^{q_{4} / q_{3}}\right)+\kappa_{3}\left|\kappa_{2}^{q_{4} / q_{3}} \xi_{2}^{q_{4}}\right|+2 \xi_{a} \omega_{a}\left|x_{3}\right|+\omega_{a}^{2}\left|x_{2}\right|+\left|z_{3}\right| \\
& \quad \leq \delta_{a} \sqrt{V}+L_{a}
\end{aligned}
$$

where $\delta_{v_{1}}, \delta_{v_{2}}, \delta_{a}, L_{v_{1}}, L_{v_{2}}, L_{a}$ are positive constants.

Considering the inequality $a b \leq a^{2} / 2+b^{2} / 2$, the following equation is easily obtained:

$$
\begin{aligned}
& \dot{V} \leq \frac{x_{1}^{2}+x_{2}^{2}}{2}+\frac{x_{1}^{2}+z_{1}^{2}}{2}+\frac{x_{1}^{2}+\sigma_{1}^{2}}{2}+\frac{x_{2}^{2}+x_{3}^{2}}{2}+2 \xi_{a} \omega_{a} x_{3}^{2}+\omega_{a}^{2} \frac{x_{2}^{2}+x_{3}^{2}}{2} \\
& \omega_{a}^{2} a_{M c}\left|x_{3}\right|+\frac{z_{0}^{2}+v_{0}^{2}}{2}+\frac{z_{0}^{2}+x_{2}^{2}}{2}+\frac{z_{1}^{2}+v_{1}^{2}}{2}+\frac{z_{2}^{2}+v_{2}^{2}}{2}+\lambda_{0} L\left|z_{3}\right|
\end{aligned}
$$

According to Lemma 1, the estimation error $\sigma_{1}$ is globally bounded, and substituting Eqs. (33) - (36) into Eq. (37) leads to:

$$
\dot{V} \leq \delta_{1} V+\delta_{2} \sqrt{V}+L_{1}
$$

where $\delta_{1}, \delta_{2}, L_{1}$ are three positive constants.

We must consider the following two cases to facilitate the proving the theorem:

Case 1. $V \geq 1$. We have $\sqrt{V} \leq V$, consequently, Eq. (38) becomes:

$$
\dot{V} \leq\left(\delta_{1}+\delta_{2}\right) V+L_{1}
$$

Case 2. $0<V<1$. In such case, $\sqrt{V}<1+V$, then, Eq. (38) becomes:

$$
\dot{V} \leq\left(\delta_{1}+\delta_{2}\right) V+\delta_{2}+L_{1}
$$


Combining the preceding two cases implies that:

$$
\dot{V} \leq \delta_{v} V+L_{v}
$$

where $\delta_{v}, L_{v}$ are two positive constants. The solution of inequality (41) in the time interval $[0, t]$ is obtained as:

$$
V \leq\left[V(0)+\frac{L_{v}}{\delta_{v}}\right] e^{\delta_{v} t}-\frac{L_{v}}{\delta_{v}}
$$

where $V(0)$ is the initial value of $V$. Eq. (42) shows that the states of system will be bounded in a finite time during the convergent phase of FTDOB.

Step 2: System (14) reduces to system (15) and guidance law (28) to (29) when $t \geq t_{r}$ because the error dynamics of FTDOB is finite-time stable and close-loop guidance system will not escape to infinite. Substituting Eq. (29) into (27) yields:

$$
\begin{aligned}
\dot{V}_{3} \leq\left(-\kappa_{1}+c_{21}+\bar{\rho}_{21}+\bar{\rho}_{31}\right)\left|\xi_{1}\right|^{2-\tau} & +\left(-\kappa_{2}+c_{22}+c_{31}+\bar{\rho}_{22}+\bar{\rho}_{32}\right)\left|\xi_{2}\right|^{2-\tau} \\
& +\left(c_{32}+\bar{\rho}_{33}\right)\left|\xi_{3}\right|^{2-\tau}-\kappa_{3}\left|\xi_{3}\right|^{2-\tau}
\end{aligned}
$$

Let

$$
\varepsilon_{1}=\kappa_{1}-\left(c_{21}+\bar{\rho}_{21}+\bar{\rho}_{31}\right) \geq 0 \quad, \quad \varepsilon_{2}=\kappa_{2}-\left(c_{22}+c_{31}+\bar{\rho}_{22}+\bar{\rho}_{32}\right) \geq 0 \quad,
$$

$\varepsilon_{3}=\kappa_{3}-\left(c_{32}+\bar{\rho}_{33}\right) \geq 0$ yields:

$$
\dot{V}_{3} \leq-\varepsilon\left(\left|\xi_{1}\right|^{2-\tau}+\left|\xi_{2}\right|^{2-\tau}+\left|\xi_{3}\right|^{2-\tau}\right)
$$

with $\varepsilon=\min \left\{\varepsilon_{1}, \varepsilon_{2}, \varepsilon_{3}\right\}$. According to Lemma 2 and similar process in [33], we obtain for $i=1,2,3$

$$
\int_{\bar{x}_{i}^{*}}^{\bar{x}_{i}}\left(s^{1 / q_{i}}-\left(\bar{x}_{i}^{*}\right)^{1 / q_{i}}\right)^{2-q_{i}} d s \leq 2^{1-q_{i}}\left|\xi_{i}\right|^{2}
$$

This implies that:

$$
V_{3} \leq 2^{1-q_{1}}\left|\xi_{1}\right|^{2}+2^{1-q_{2}}\left|\xi_{2}\right|^{2}+2^{1-q_{3}}\left|\xi_{3}\right|^{2} \leq \bar{\kappa}\left(\left|\xi_{1}\right|^{2}+\left|\xi_{2}\right|^{2}+\left|\xi_{3}\right|^{2}\right)
$$

where $\bar{\kappa}=\max \left(2^{1-q_{1}}, 2^{1-q_{2}}, 2^{1-q_{3}}\right)$ is a constant. Together with Lemma 4 yields:

$$
\left|\xi_{1}\right|^{2-\tau}+\left|\xi_{2}\right|^{2-\tau}+\left|\xi_{3}\right|^{2-\tau}=\left(\xi_{1}^{2}\right)^{(2-\tau) / 2}+\left(\xi_{2}^{2}\right)^{(2-\tau) / 2}+\left(\xi_{3}^{2}\right)^{(2-\tau) / 2} \geq \bar{\kappa}^{-(2-\tau) / 2} V_{3}^{(2-\tau) / 2}
$$

Hence,

$$
\dot{V}_{3}+\bar{\kappa}^{-(2-\tau) / 2} V_{3}^{(2-\tau) / 2} \leq(1-\varepsilon)\left(\left|\xi_{1}\right|^{2-\tau}+\left|\xi_{2}\right|^{2-\tau}+\left|\xi_{3}\right|^{2-\tau}\right)
$$

According to Lemma 1, if $\varepsilon \geq 1$, the closed-loop guidance system is finite-time stable. Therefore, the LOS angular rate will converge to zero in finite time and control objective is achieved by invoking the definition of $\xi_{i}$.

\section{Numerical simulations}

\section{Simulations with varying missile velocity}

In this section, numerical simulations are performed to demonstrate the efficacy of the proposed guidance law for various kinds of target maneuvers. Suppose the missile 
is equipped with an active radar seeker, providing LOS angle, LOS angular rate, range, and range rate information.

Although the engagement model is constructed with consideration that both the missile and the target velocities are constant, guidance law (29) also performs well with varying speeds. For simplicity, only varying missile velocity considered here. Under this condition, Eq. (6) can be rewritten as:

$$
\dot{V}_{q}=-\frac{V_{r} V_{q}}{r}+a_{T q}-a_{M q}+\dot{V}_{M} \sin \left(q-\gamma_{M}\right)
$$

Define $h=-\frac{V_{r} V_{q}}{r}+a_{T q}-a_{M q}+a_{M}+\dot{V}_{M} \sin \left(q-\gamma_{M}\right)$ as the new lumped uncertainty; substituting into Eq. (49) yields:

$$
\dot{V}_{q}=h-a_{M}
$$

which has the same structure as shown in Eq. (8). Therefore, guidance law (29) still works well if lumped uncertainty $h$ is estimated accurately.

A more realistic intercept model [34] is presented by considering effects of thrust and aerodynamics. Missile acceleration is normal in missile velocity, and the dynamics of missile velocity can be given in body frame as:

$$
\dot{V}_{M}=\frac{T-D}{M}
$$

where $T$ is the thrust, $D$ is the drag force, and $M$ is its mass. The thrust profile is given by:

$$
T= \begin{cases}T_{0}, & t \leq t_{b} \\ 0, & t>t_{b}\end{cases}
$$

The mass of the missile changes when propulsion system is being on, and it can be approximated as:

$$
M=m_{i}-\frac{m_{p}}{t_{b}} t
$$

where $m_{i}, m_{p}$ is the initial mass of missile and mass of propellant, respectively. The term $m_{p} / t_{b}$ represents fuel mass flow rate, where $t_{b}$ is the burn time.

The profile of drag force is governed by:

$$
D=\frac{1}{2} \rho V_{M}^{2} C_{D} A
$$

where $\rho$ is the atmosphere air density, $A$ denotes the reference area and $C_{D}$ is the drag force coefficient. $C_{D}$ is given by a parabolic model as $C_{D}=C_{D 0}+k C_{L}^{2} ; C_{D 0}$ and $C_{L}$ denote zero lift drag coefficient and lift force coefficient, respectively, and $k$ 
is the parameter of induced drag. As the lift force acting on the missile is given as $L=0.5 \rho V_{M}^{2} C_{L}$ and lateral acceleration is $a_{c}=L / M$, the resulting lift coefficient is expressed in $C_{L}=2 a_{M} M /\left(\rho V_{M}^{2} A\right)$.

The initial engagement condition and related data required in closed system (11) are selected as: 1) initial missile-target relative distance: $14142 \mathrm{~m}$; 2) initial LOS angle: $\pi / 4 \mathrm{rad} ; 3$ ) initial flight path angle of missile: $\pi / 4 \mathrm{rad}$; 4) initial flight path angle of target: $\pi$ rad; 5) initial missile velocity: $800 \mathrm{~m} / \mathrm{s} ; 6$ ) initial target velocity: $500 \mathrm{~m} / \mathrm{s}$; 7) autopilot parameters: $\left.\xi_{a}=0.8, \omega_{a}=10 \mathrm{rad} / \mathrm{s} ; 8\right) \max$ acceleration of missile achieved: $200 \mathrm{~m} / \mathrm{s}^{2}$; 9) parameters of thruster: $m_{i}=165 \mathrm{~kg}, m_{p}=15 \mathrm{~kg}, t_{b}=5 \mathrm{~s}$, and $T_{0}=17640 \mathrm{~N}$; and 10) parameters of aerodynamic force: $C_{D 0}=0.74, k=0.03$, $A=0.0324 \mathrm{~m}^{2}$, and $\rho=0.909 \mathrm{~kg} / \mathrm{m}^{3}$. Three different target maneuver profiles are given below for simulations.

Case 1: Constant target maneuvers $a_{T}=+80 \mathrm{~m} / \mathrm{s}^{2}$.

Case 2: Sudden evasive target maneuvers $a_{T}=0 \mathrm{~m} / \mathrm{s}^{2}$ for $t<2 \mathrm{~s}, a_{T}=-100 \mathrm{~m} / \mathrm{s}^{2}$ for $2<t<4 \mathrm{~s}$, and $a_{T}=100 \mathrm{~m} / \mathrm{s}^{2}$ for $t>4 \mathrm{~s}$.

Case 3: Periodic target maneuvers $a_{T}=-100 \sin (2 t) \mathrm{m} / \mathrm{s}^{2}$.

The design parameters for implementing proposed guidance law (29) are set as $k_{1}=5, k_{2}=20, k_{3}=40$, and $\tau=2 / 39$. The selection of observer gains of FTDOB is given as: $\lambda_{0}=1.1, \lambda_{1}=1.5, \lambda_{2}=5$, and $\lambda_{3}=10$.

For making a better showcase, the adaptive sliding mode guidance law (ASMG) [11] and finite time convergent guidance law (FTCG) [17] are also considered in the simulation for comparison.

The ASMG law is defined as:

$$
a_{M c}=-N_{a} V_{r} \dot{q}+C \dot{q} /(|\dot{q}|+\delta)
$$

where the effective navigation ratio $N_{a}$ is usually $3-5$, and $C>0$ is a design parameter. The parameters are selected as, $N_{a}=4$ and $C=100$. The FTCG law is defined as:

$$
a_{M c}=-N_{f} V_{r} \dot{q}+\beta_{1} \operatorname{sgn}(\dot{q})+\beta_{2}|\dot{q}|^{\eta} \operatorname{sgn}(\dot{q})
$$

where $N_{f}>2, \beta_{1}, \beta_{2}>0$, and $0<\eta<1$ are design parameters and selected as $N_{f}=4, \beta_{1}=100, \beta_{2}=30$, and $\eta=0.5$. However, the undesired violent chattering phenomenon will result from the property of discontinuous terms $\beta_{1} \operatorname{sgn}(\dot{q})$. A saturation function $\operatorname{sat}(x)$ is used to replace the sign function to avoid this phenomenon, where:

$$
\operatorname{sat}(x)=\left\{\begin{array}{c}
\operatorname{sgn}(x),|x|>\eta \\
x / \eta,|x| \leq \eta
\end{array}\right.
$$

where $\eta$ is a small positive constant and is set as 0.005 in simulations. 


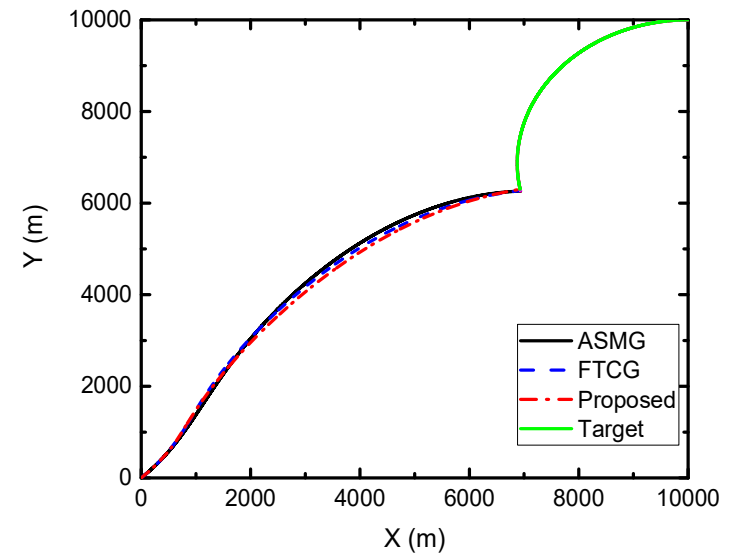

(a)

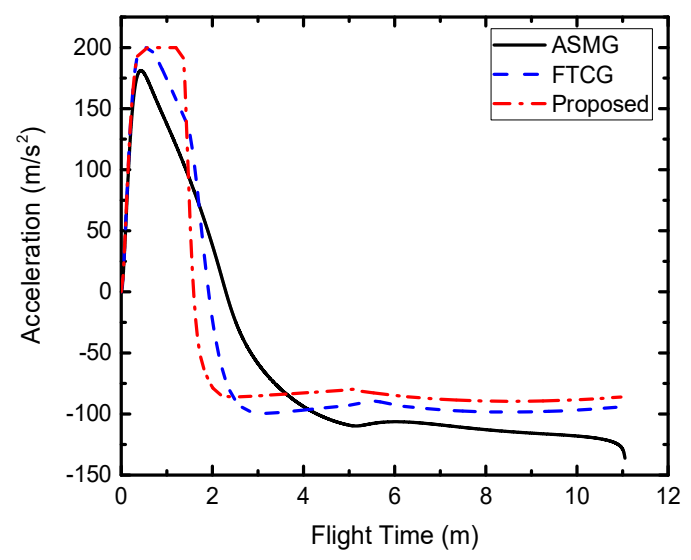

(c)

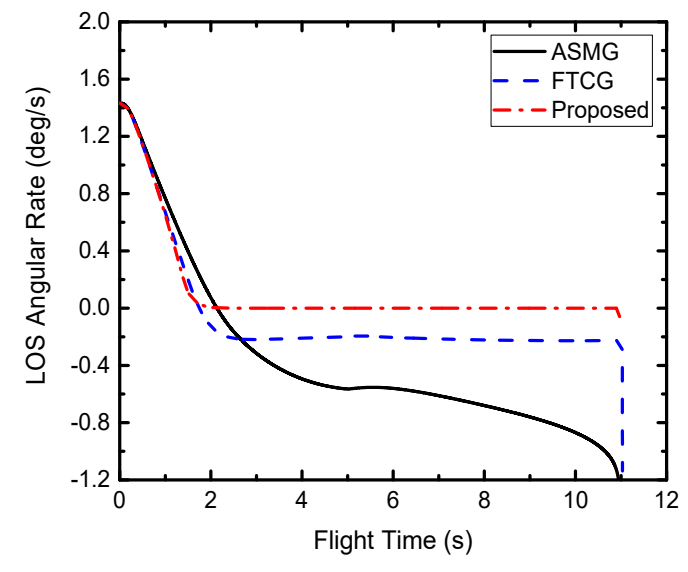

(b)

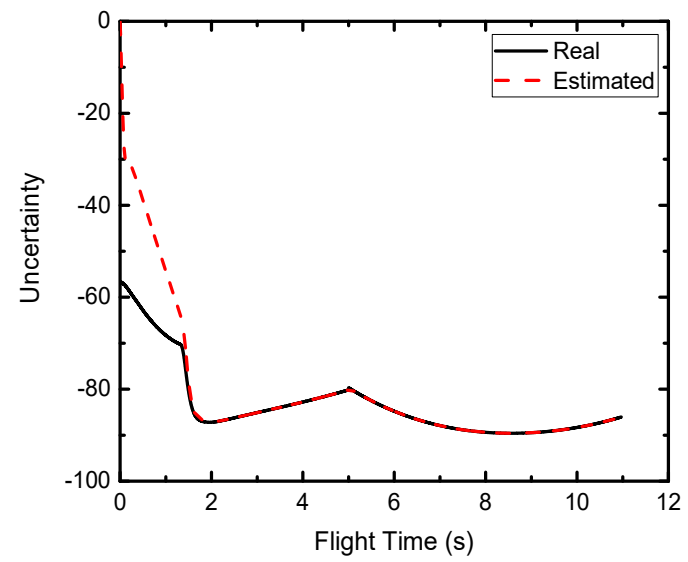

(d)

Fig. 2. Results of comparison under three guidance laws for case 1: (a) interception trajectories;

(b) LOS angular rates; (c) acceleration of missile achieved; and (d) uncertainty estimations.

The simulations are carried out for three different target acceleration profiles and the results of comparison are presented in the following figures: Fig. 2 is for Case 1; Fig. 3 is for Case 2; and Fig. 4 is for Case 3. The figures contain profiles of interception trajectories, LOS angular rates, acceleration of missile achieved, and uncertainty estimations. The proposed guidance law has offered better performance in all cases compared with other guidance laws. The LOS angular rate is regulated to zero rapidly in finite time under proposed guidance law, whereas ASMG only keeps the LOS rate boundness and becomes divergence as close to target. As a result of initial error in estimation, the required acceleration of missile for proposed law is higher initially. However, acceleration profile is maintained more practically acceptable than other laws in most of flight time. 


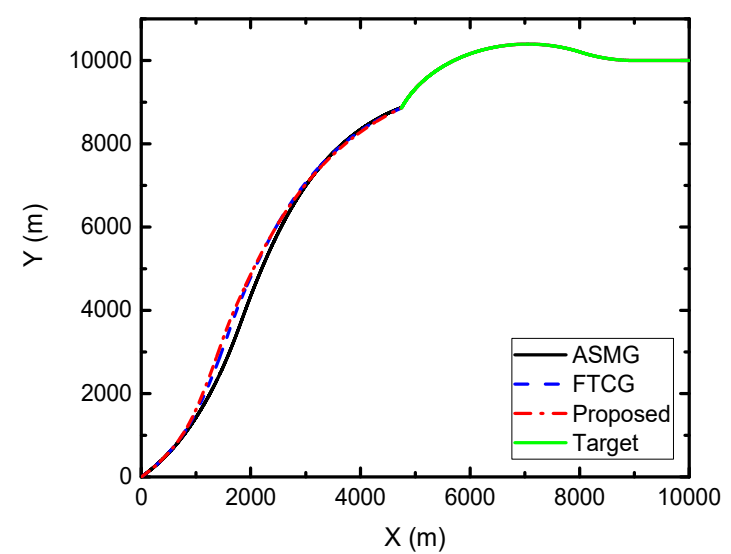

(a)

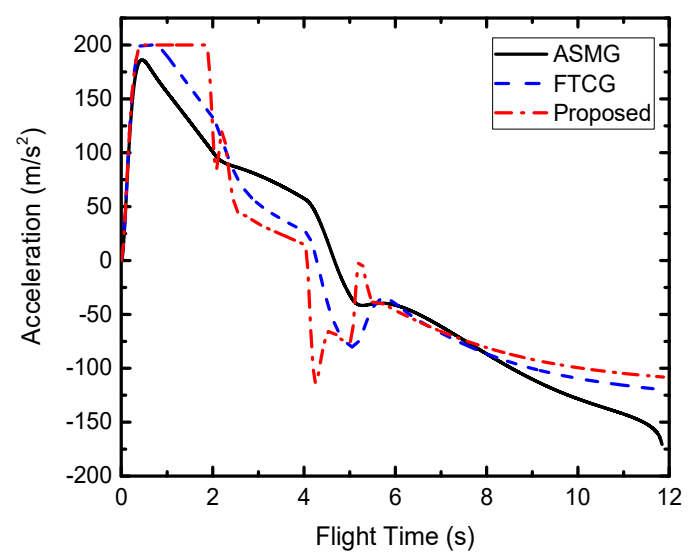

(c)

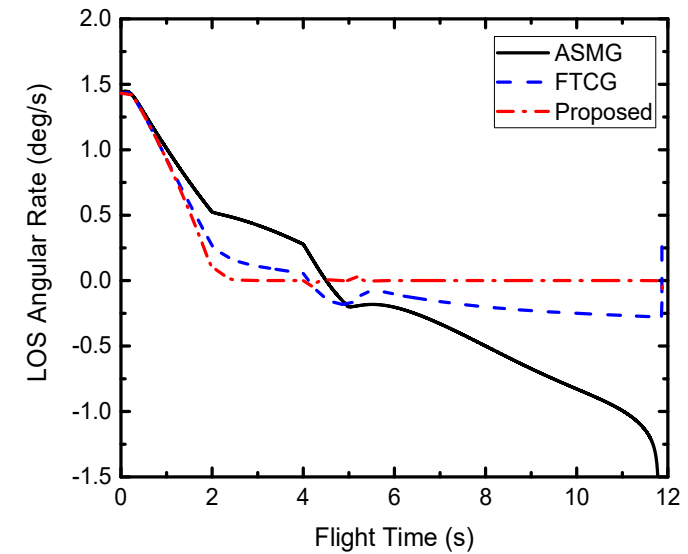

(b)

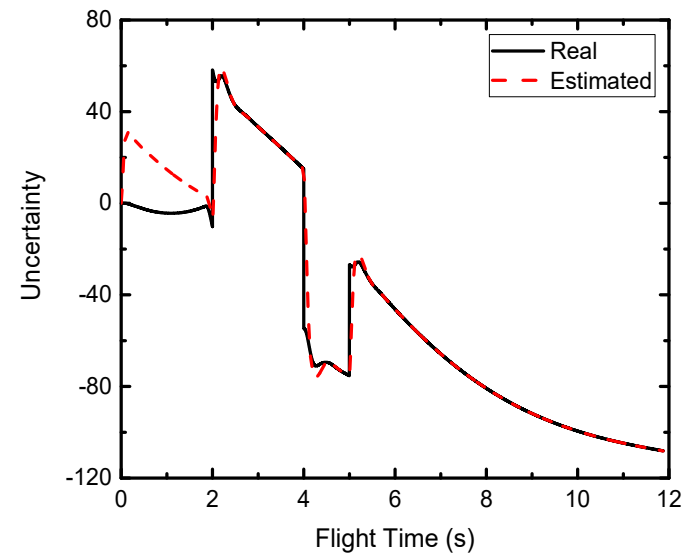

(d)

Fig. 3. Results of comparison under three guidance laws for case 2: (a) interception trajectories;

(b) LOS angular rates; (c) acceleration of missile achieved; and (d) uncertainty estimations.

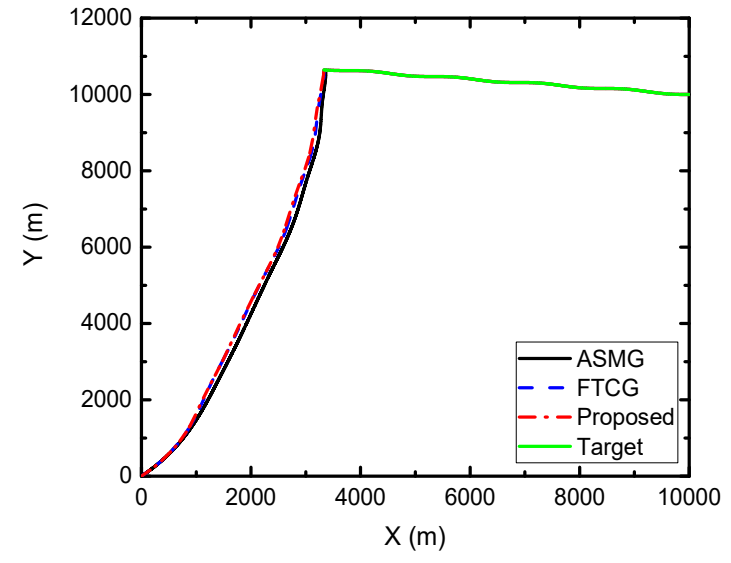

(a)

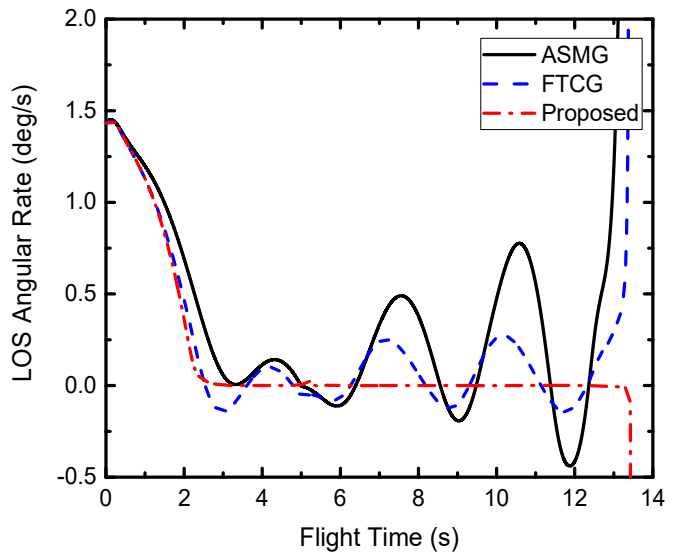

(b) 


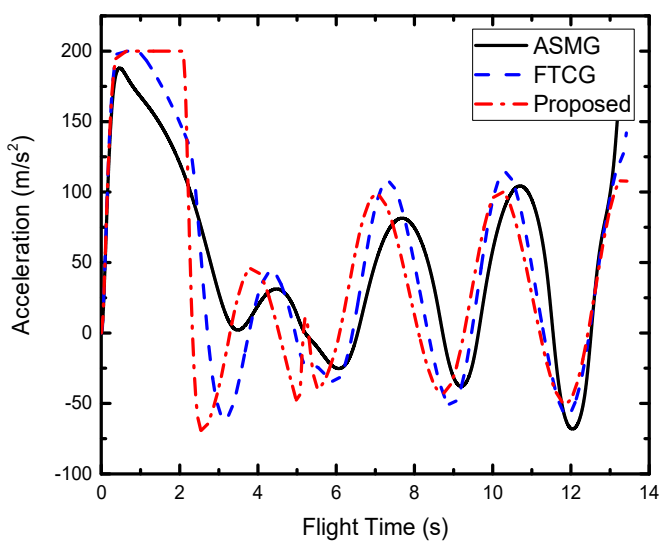

(c)

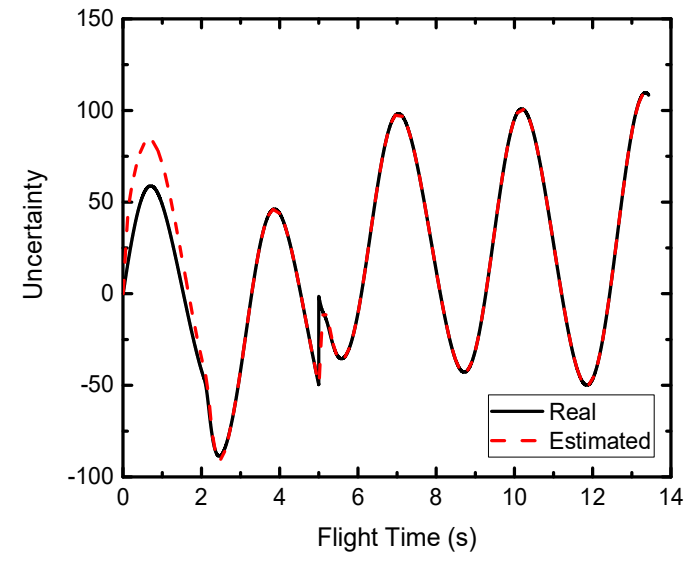

(d)

Fig. 4. Results of comparison under three guidance laws for case 3: (a) interception trajectories;

(b) LOS angular rates; (c) acceleration of missile achieved; and (d) uncertainty estimations.

Additionally, FTCG law can drive LOS angular rate around zero in finite time, whereas the LOS rate cannot converge to a small region. The reason is that the chattering problem is addressed when FTCG law has adopted boundary layer technique. Thus, only motion around the sliding surface can be maintained. The chattering behavior of LOS angular rate under ASMG and FTCG laws are more apparent than proposed law in Case 3, which is not desirable for seeker system, because of the existence of missile autopilot lag. In contrast, with effective autopilot lag compensation, the proposed guidance law displays better transient performance and attains steady state earlier. The miss distances and interception time obtained by these considered guidance laws under target acceleration cases are as given in Table I. From the table it can be noted that the interception time spent by ASMG, FTCG and proposed laws is similar for all target situations. However, the miss distances achieved by proposed law are much more less than other laws, the reason is that the proposed law can keep the LOS angular rate to zero during most of the flight time.

Table I. Miss Distances and interception time

\begin{tabular}{|c|c|c|c|c|c|c|}
\hline \multirow{2}{*}{$\begin{array}{c}\text { Guidance } \\
\text { Laws }\end{array}$} & \multicolumn{2}{|c|}{ Case 1} & \multicolumn{2}{|c|}{ Case 2} & \multicolumn{2}{|c|}{ Case 3} \\
\hline & $\begin{array}{c}\text { Miss Distance } \\
(\mathrm{m})\end{array}$ & $t_{f}(\mathrm{~s})$ & $\begin{array}{c}\text { Miss Distance } \\
(\mathrm{m})\end{array}$ & $t_{f}(\mathrm{~s})$ & $\begin{array}{c}\text { Miss Distance } \\
(\mathrm{m})\end{array}$ & $t_{f}(\mathrm{~s})$ \\
\hline ASMG & 0.0781 & 11.028 & 0.0519 & 11.824 & 0.8095 & 13.337 \\
\hline FTCG & 0.0019 & 11.008 & 0.0018 & 11.852 & 0.0438 & 13.390 \\
\hline Proposed & $9 \times 10^{-6}$ & 10.947 & $7 \times 10^{-6}$ & 11.861 & $4 \times 10^{-4}$ & 13.407 \\
\hline
\end{tabular}

\section{Simulations with measurement noise}

\begin{tabular}{cc}
\multicolumn{2}{c}{ Table II. Measurement noise } \\
\hline Parameter & $\begin{array}{c}\text { Standard } \\
\text { deviation }\end{array}$ \\
\hline$\dot{q}(\mathrm{rad} / \mathrm{s})$ & $5 \times 10^{-5}$ \\
\hline$r(\mathrm{~m})$ & 30.48 \\
\hline
\end{tabular}


The information of sensors is always affected by the noise in realistic scenario, which leads to degrade the performance of closed-loop guidance system [35], especially for the FTDOB. Simulations are carried out to test the performance of proposed guidance law by considering corrupted measurements of LOS angular rate and range. Measurement noises are assumed to be normally distributed with zero mean and corresponding standard deviations are given by Table II [36].

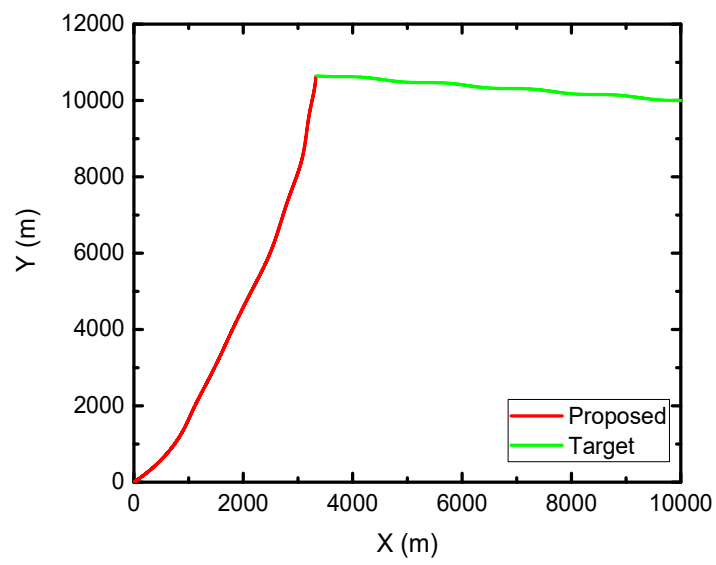

(a)

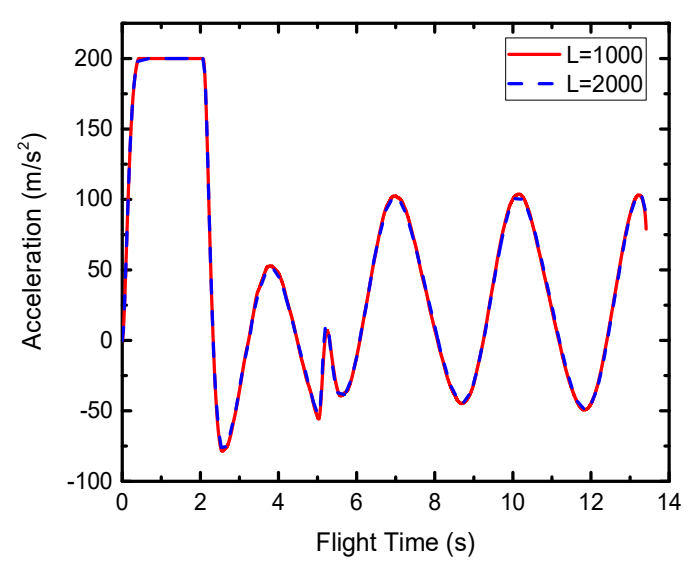

(c)

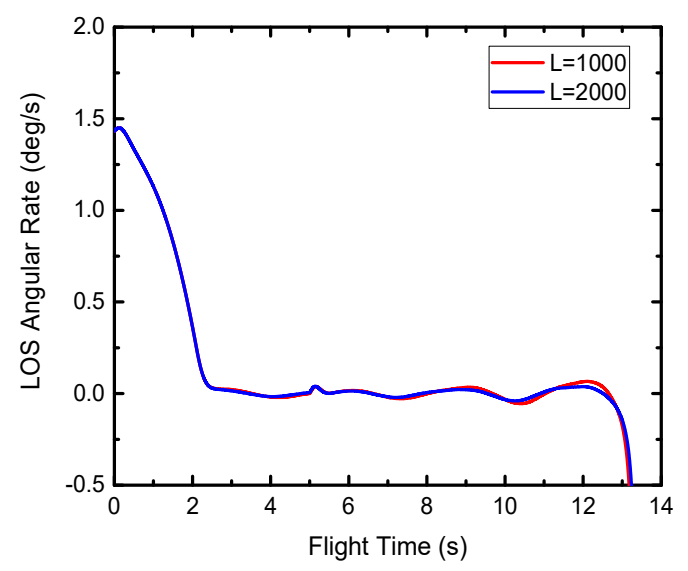

(b)

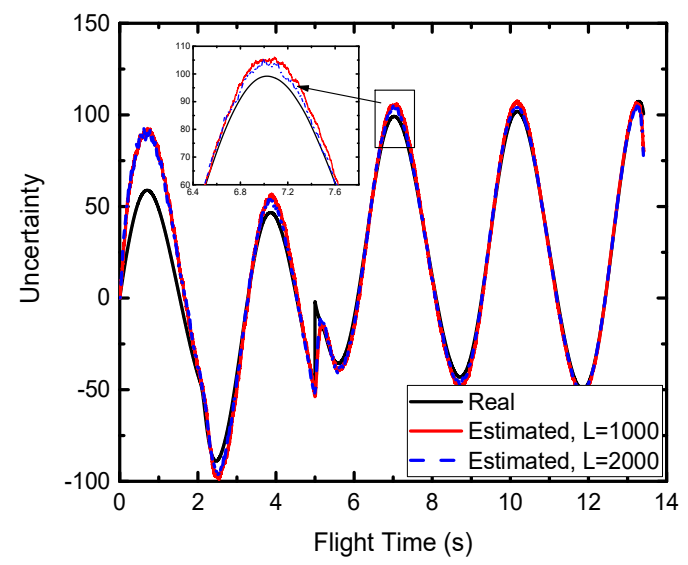

(d)

Fig. 5. Performance of proposed guidance law in noise-corrupted environment for case 3: (a) interception trajectories; (b) LOS angular rates; (c) acceleration of missile achieved; and (d) uncertainty estimations.

Table III. Miss Distances and interception time

\begin{tabular}{|c|c|c|}
\hline \multirow[b]{2}{*}{ Guidance Laws } & \multicolumn{2}{|c|}{ Case 3} \\
\hline & $\begin{array}{c}\text { Miss Distance } \\
(\mathrm{m})\end{array}$ & $t_{f}(\mathrm{~s})$ \\
\hline Proposed $L=1000$ & 0.2926 & 13.4130 \\
\hline Proposed $L=2000$ & 0.1559 & 13.4097 \\
\hline
\end{tabular}

The simulation results in noisy environment for Case 3 are presented in Fig. 5. From Fig. 5(d), it can be observed that the lumped uncertainty can be well estimated through FTDOB and estimation error remains bounded, even if there exist measurement noises. 
The performance of FTDOB can improve slightly, when the gain of FTDOB $L$ is set as twice as the original one. Moreover, the LOS angular rate also regulated to a small region around zero and missile engages target accurately notwithstanding target maneuver. Table III shows the corresponding mean miss distances and interception time of 100 runs Monte-Carlo simulations. The noise insensitive property may make the proposed guidance law suitable for practice use.

\section{Conclusion}

In this work, a nonlinear finite time guidance law considering second-order autopilot lag is presented for maneuvering targets. Based on finite-time backstepping control technique and FTDOB method, the guidance law can guarantee that LOS angular rate converges to zero in finite time. In the design, the guidance law is derived by finitetime backstepping method and lumped uncertainty can be estimated incorporating with FTDOB. The time derivatives of virtual controls are avoided by applying integral-type Lyapunov functions. Simulations and comparisons of various engagement scenarios demonstrate the excellent interception provided by proposed guidance law with autopilot dynamics.

\section{Conflict of interest statement}

The authors declare that no conflict of interest exists regarding the publication of this article.

\section{Appendix. Proof for key propositions}

Proof of Proposition 2:

The following equation can be concluded from Proposition 1:

$$
\left|\frac{\partial W_{2}}{\partial \bar{x}_{1}} \dot{\bar{x}}_{1}\right| \leq\left(2-q_{2}\right)\left|\bar{x}_{2}-\bar{x}_{2}^{*}\right|\left|\xi_{2}\right|^{1-q_{2}}\left|\frac{\partial\left(\bar{x}_{2}^{*}\right)^{1 / q_{2}}}{\partial \bar{x}_{1}} \dot{\bar{x}_{1}}\right|
$$

By using Lemma 2 and derivation in [33], we have following inequality:

$$
\left|\bar{x}_{k}-\bar{x}_{k}^{*}\right| \leq 2\left|\xi_{k}\right|^{q_{k}}
$$

Then, substituting (59) into (58) gives:

$$
\left|\frac{\partial W_{2}}{\partial \bar{x}_{1}} \dot{\overline{x_{1}}}\right| \leq a_{2}\left|\xi_{2}\right|\left|\frac{\partial\left(\bar{x}_{2}^{*}\right)^{1 / q_{2}}}{\partial \bar{x}_{1}} \dot{\bar{x}}_{1}\right|
$$

where $a_{2}=2 \times\left(2-q_{2}\right)$ is a positive constant.

From the definition of $\bar{x}_{2}^{*}=-\kappa_{1} \xi_{1}^{q_{2}}, \quad \xi_{1}=\bar{x}_{1}^{1 / q_{1}}=\bar{x}_{1}$, we have:

$$
\left|\frac{\partial\left(\bar{x}_{2}^{*}\right)^{1 / q_{2}}}{\partial \bar{x}_{1}} \dot{\bar{x}}\right|=\kappa_{1}^{\frac{1}{q_{2}}}\left|\bar{x}_{2}\right|
$$

and inequality $\left|\bar{x}_{2}\right| \leq\left(\left|\xi_{2}\right|^{q_{2}}+\kappa_{1}\left|\xi_{1}\right|^{q_{2}}\right)$ according to Lemma 4. Combining Eq. (61) and Lemma 3, we have: 


$$
\begin{aligned}
& \left|\frac{\partial W_{2}}{\partial \bar{x}_{1}} \dot{\bar{x}}\right| \leq a_{2} \kappa_{1}^{\frac{1}{q_{2}}}\left|\xi_{2}\right|\left(\left|\xi_{2}\right|^{q_{2}}+\kappa_{1}\left|\xi_{1}\right|^{q_{2}}\right) \\
& \leq a_{2} \kappa_{1}^{\frac{1}{q_{2}}}\left|\xi_{2}\right|^{2-\tau}+a_{2} \kappa_{1}^{\frac{1}{q_{2}}+1}\left(\frac{1}{1+q_{2}}\left|\xi_{1}\right|^{2-\tau}+\frac{q_{2}}{1+q_{2}}\left|\xi_{2}\right|^{2-\tau}\right) \\
& \leq \bar{\rho}_{21}\left|\xi_{1}\right|^{2-\tau}+\bar{\rho}_{22}\left|\xi_{2}\right|^{2-\tau}
\end{aligned}
$$

where $\bar{\rho}_{21}>0$ and $\bar{\rho}_{22}>0$ are positive constants. This completes the proof. Proof of Proposition 3:

The following equation can be obtained from Proposition 1 and Lemma 2:

$$
\left|\frac{\partial W_{3}}{\partial \bar{x}_{1}} \dot{\bar{x}}_{1}+\frac{\partial W_{3}}{\partial \bar{x}_{2}} \dot{\bar{x}}_{2}\right| \leq\left(2-q_{3}\right)\left|\bar{x}_{3}-\bar{x}_{3}^{*}\right|\left|\xi_{3}\right|^{1-q_{3}}\left|\frac{\partial\left(\bar{x}_{3}^{*}\right)^{1 / q_{3}}}{\partial \bar{x}_{1}} \dot{\bar{x}}_{1}+\frac{\partial\left(\bar{x}_{3}^{*}\right)^{1 / q_{3}}}{\partial \bar{x}_{2}} \dot{\bar{x}}_{2}\right|
$$

With similar process in proof of Proposition 2, we have:

$$
\left|\frac{\partial W_{3}}{\partial \bar{x}_{1}} \dot{\bar{x}}_{1}+\frac{\partial W_{3}}{\partial \bar{x}_{2}} \dot{\bar{x}}_{2}\right| \leq a_{3}\left|\xi_{3}\right|\left|\frac{\partial\left(\bar{x}_{3}^{*}\right)^{1 / q_{3}}}{\partial \bar{x}_{1}} \dot{\bar{x}}_{1}+\frac{\partial\left(\bar{x}_{3}^{*}\right)^{1 / q_{3}}}{\partial \bar{x}_{2}} \dot{\bar{x}}_{2}\right|
$$

where $a_{3}=2 \times\left(2-q_{3}\right)$ is a positive constant.

Note that $\bar{x}_{3}^{*}=-\kappa_{2} \xi_{2}^{q_{3}}$ and $\xi_{2}=\bar{x}_{2}^{1 / q_{2}}-\left(\bar{x}_{2}^{*}\right)^{1 / q_{2}}$, showing that:

$$
\begin{gathered}
\left|\frac{\partial\left(\bar{x}_{3}^{*}\right)^{1 / q_{3}}}{\partial \bar{x}_{1}} \dot{\dot{x}_{1}}\right|=\kappa_{2}^{\frac{1}{q_{3}}}\left|\frac{\partial \xi_{2}}{\partial \bar{x}_{1}} \dot{\overline{x_{1}}}\right| \leq \kappa_{2}^{\frac{1}{q_{3}}}\left|\frac{\partial\left(\bar{x}_{2}^{*}\right)^{1 / q_{2}}}{\partial \bar{x}_{1}}\right|\left|\bar{x}_{2}\right| \\
\left|\frac{\partial\left(\bar{x}_{3}^{*}\right)^{1 / q_{3}}}{\partial \bar{x}_{2}} \dot{\bar{x}}_{2}\right|=\kappa_{2}^{\frac{1}{q_{3}}}\left|\frac{\partial \xi_{2}}{\partial \bar{x}_{2}} \dot{\bar{x}}_{2}\right| \leq \kappa_{2}^{\frac{1}{q_{3}}}\left|\frac{\partial\left(\bar{x}_{2}\right)^{1 / q_{2}}}{\partial \bar{x}_{2}}\right|\left|\bar{x}_{3}\right| \leq \frac{\kappa_{2}^{\frac{1}{q_{3}}}}{q_{2}}\left|\bar{x}_{2}\right|^{\frac{1-q_{2}}{q_{2}}}\left|\bar{x}_{3}\right|
\end{gathered}
$$

From Eq. (61) and inequalities $\left|\bar{x}_{2}\right| \leq\left(\left|\xi_{2}\right|^{q_{2}}+\kappa_{1}\left|\xi_{1}\right|^{q_{2}}\right), \quad\left|\bar{x}_{3}\right| \leq\left(\left|\xi_{3}\right|^{q_{3}}+\kappa_{2}\left|\xi_{2}\right|^{q_{3}}\right)$, we have the following:

$$
\begin{gathered}
\left|\frac{\partial\left(\bar{x}_{3}^{*}\right)^{1 / q_{3}}}{\partial \bar{x}_{1}} \dot{\overline{x_{1}}}\right| \leq \kappa_{1}^{\frac{1}{q_{2}}} \kappa_{2}^{\frac{1}{q_{3}}}\left|\bar{x}_{2}\right| \leq \gamma_{1}\left(\left|\xi_{2}\right|^{q_{2}}+\left|\xi_{1}\right|^{q_{2}}\right) \\
\left|\frac{\partial\left(\bar{x}_{3}^{*}\right)^{1 / q_{3}}}{\partial \bar{x}_{2}} \dot{\bar{x}}_{2}\right| \leq \frac{\kappa_{2}^{\frac{1}{q_{3}}}}{q_{2}}\left|\bar{x}_{2}\right|^{\frac{1-q_{2}}{q_{2}}}\left|\bar{x}_{3}\right| \leq \frac{\kappa_{2}^{\frac{1}{q_{3}}}}{q_{2}}\left(\left|\xi_{2}\right|^{q_{2}}+\kappa_{1}\left|\xi_{1}\right|^{q_{2}}\right)^{\frac{1-q_{2}}{q_{2}}}\left|\bar{x}_{3}\right| \\
\leq \frac{\kappa_{2}^{\frac{1}{q_{3}}}}{q_{2}}\left(\left|\xi_{2}\right|^{1-q_{2}}+\kappa_{1}^{\frac{1-q_{2}}{q_{2}}}\left|\xi_{1}\right|^{1-q_{2}}\right)\left(\left|\xi_{3}\right|^{q_{3}}+\kappa_{2}\left|\xi_{2}\right|^{q_{3}}\right) \leq \gamma_{2}\left(\left|\xi_{1}\right|^{q_{2}}+\left|\xi_{2}\right|^{q_{2}}+\left|\xi_{1}\right|^{q_{2}}\right)
\end{gathered}
$$

where the last inequality is obtained using Lemma 3 and $4, \gamma_{1}>0$ and $\gamma_{2}>0$ are positive constants.

Considering Eq. (67), (68), and Lemma 3, we have the following: 


$$
\begin{aligned}
& \left|\frac{\partial W_{3}}{\partial \bar{x}_{1}} \dot{\bar{x}}_{1}+\frac{\partial W_{3}}{\partial \bar{x}_{2}} \dot{\bar{x}}_{2}\right| \leq a_{3} \gamma_{1}\left|\xi_{3}\right|\left(\left|\xi_{1}\right|^{q_{2}}+\left|\xi_{2}\right|^{q_{2}}\right)+a_{3} \gamma_{2}\left|\xi_{3}\right|\left(\left|\xi_{1}\right|^{q_{2}}+\left|\xi_{2}\right|^{q_{2}}+\left|\xi_{3}\right|^{q_{2}}\right) \\
& \leq a_{3} \gamma_{1}\left(\frac{1}{1+q_{2}}\left|\xi_{3}\right|^{1+q_{2}}+\frac{q_{2}}{1+q_{2}}\left|\xi_{1}\right|^{1+q_{2}}\right)+a_{3} \gamma_{1}\left(\frac{1}{1+q_{2}}\left|\xi_{3}\right|^{1+q_{2}}+\frac{q_{2}}{1+q_{2}}\left|\xi_{2}\right|^{1+q_{2}}\right) \\
& +a_{3} \gamma_{2}\left(\frac{1}{1+q_{2}}\left|\xi_{3}\right|^{1+q_{2}}+\frac{q_{2}}{1+q_{2}}\left|\xi_{1}\right|^{1+q_{2}}\right)+a_{3} \gamma_{2}\left(\frac{1}{1+q_{2}}\left|\xi_{3}\right|^{1+q_{2}}+\frac{q_{2}}{1+q_{2}}\left|\xi_{2}\right|^{1+q_{2}}\right)+a_{3} \gamma_{2}\left|\xi_{3}\right|^{1+q_{2}} \\
& \leq \bar{\rho}_{31}\left|\xi_{1}\right|^{1+q_{2}}+\bar{\rho}_{32}\left|\xi_{2}\right|^{1+q_{2}}+\bar{\rho}_{33}\left|\xi_{3}\right|^{1+q_{2}}=\bar{\rho}_{31}\left|\xi_{1}\right|^{2-\tau}+\bar{\rho}_{32}\left|\xi_{2}\right|^{2-\tau}+\bar{\rho}_{33}\left|\xi_{3}\right|^{2-\tau}
\end{aligned}
$$

where $\bar{\rho}_{31}>0, \bar{\rho}_{32}>0$, and $\bar{\rho}_{33}>0$ are positive constants. This completes the proof.

\section{References:}

[1].Zarchan P. Tactical and Strategic Missile Guidance, Sixth Edition[M]. American Institute of Aeronautics \& Astronautics Inc, 2012(6):555.

[2]. Ghawghawe S N, Ghose D. Pure proportional navigation against time-varying target maneuvers[J]. Aerospace \& Electronic Systems IEEE Transactions on, 1996, 32(4):1336-1347.

[3]. Yang C D, Yang C C. A unified approach to proportional navigation[J]. IEEE Transactions on Aerospace \& Electronic Systems, 1997, 33(2):557-567.

[4]. Yang C D, Chen H Y. Nonlinear Hळ Robust Guidance Law for Homing Missiles[J]. Journal of Guidance Control and Dynamics, 1998, 21(6):882-890.

[5]. Lechevin N, Rabbath C A. Lyapunov-Based Nonlinear Missile Guidance[J]. Journal of Guidance Control and Dynamics, 2012, 27(6):1096-1101.

[6]. Zhou D, Mu C, Shen T. Robust Guidance Law with L2 Gain Performance[J]. Transactions of the Japan Society for Aeronautical \& Space Sciences, 2005, 44(144):82-88.

[7]. Zhang P, Fang Y, Zhang F, et al. An Adaptive Weighted Differential Game Guidance Law[J]. Chinese Journal of Aeronautics, 2012, 25(5):739-746.

[8]. Shima T, Golan O M. Linear Quadratic Differential Games Guidance Law for Dual Controlled Missiles[J]. IEEE Transactions on Aerospace \& Electronic Systems, 2007, 43(3):834-842.

[9]. Brierley S D, Longchamp R. Application of sliding-mode control to air-air interception problem[J]. IEEE Transactions on Aerospace \& Electronic Systems, 1990, 26(2):306-325.

[10]. Babu K R, Sarma I G, Swamy K N. Switched bias proportional navigation for homing guidance against highly maneuvering targets[J]. Journal of guidance control and dynamics, 1994, 17(6):1357-1363. [11]. Zhou D, Mu C, Xu W. Adaptive Sliding-Mode Guidance of a Homing Missile[J]. Journal of Guidance Control and Dynamics, 1999, 22(4):589-594.

[12]. Moon J, Kim K, Kim Y. Design of Missile Guidance Law via Variable Structure Control[J]. Journal of Guidance Control and Dynamics, 2001, 24(4):659-664.

[13]. Yang J, Li S, Su J, et al. Continuous nonsingular terminal sliding mode control for systems with mismatched disturbances is [J]. Automatica, 2013, 49(7):2287-2291.

[14]. Yang J, Li S, Sun C, et al. Nonlinear-Disturbance-Observer-Based Robust Flight Control for Airbreathing Hypersonic Vehicles[J]. Aerospace \& Electronic Systems IEEE Transactions on, 2013, 
49(2):1263-1275.

[15]. Zhang Z, Man C, Li S, et al. Finite-time guidance laws for three-dimensional missile-target interception[J]. Proceedings of the Institution of Mechanical Engineers Part G Journal of Aerospace Engineering, 2015, 230(2).

[16]. Zhang Z, Li S, Luo S. Terminal guidance laws of missile based on ISMC and NDOB with impact angle constraint[J]. Aerospace Science \& Technology, 2013, 31(1):30-41.

[17]. Zhou D, Sun S, Teo K L, et al. Guidance Laws with Finite Time Convergence[J]. Journal of Guidance Control and Dynamics, 2009, 32(6).

[18]. Kumar S R, Rao S, Ghose D. Nonsingular Terminal Sliding Mode Guidance with Impact Angle Constraints[J]. Journal of Guidance Control and Dynamics, 2014, 37(4):1114-1130.

[19]. Golestani M, Mohammadzaman I, Vali A R. Finite-time convergent guidance law based on integral backstepping control[J]. Aerospace Science \& Technology, 2014, 39:370-376.

[20]. Sun S, Zhou D, Hou W. A guidance law with finite time convergence accounting for autopilot lag[J]. Aerospace Science \& Technology, 2013, 25(1):132-137.

[21]. Rusnak I, Meir L. Modern guidance law for high-order autopilot[J]. Journal of guidance control and dynamics, 2015, 14(5):1056-1058.

[22]. He S, Wang W, Lin D. Adaptive Backstepping impact angle guidance law accounting for autopilot lag[J]. Journal of Aerospace Engineering, 2016, 30(3):04016094.

[23]. Wu L, Wang W, Xiong S. Guidance law accounting for second-order dynamics of missile autopilot and impact angle constraints[C]// Control and Decision Conference. IEEE, 2014:2459-2464.

[24]. He S, Lin D, Wang J. Robust terminal angle constraint guidance law with autopilot lag for intercepting maneuvering targets[J]. Nonlinear Dynamics, 2015, 81(1-2):881-892.

[25]. Zhou D, Qu P, Sun S. A Guidance Law With Terminal Impact Angle Constraint Accounting for Missile Autopilot[J]. Journal of Dynamic Systems Measurement \& Control, 2013, 135(5):051009.

[26]. He S, Wang W, Wang J. Adaptive backstepping impact angle control with autopilot dynamics and acceleration saturation consideration[J]. International Journal of Robust \& Nonlinear Control, 2017.

[27]. He S, Song T, Lin D. Impact Angle Constrained Integrated Guidance and Control for Maneuvering Target Interception[J]. Journal of Guidance Control and Dynamics, 2017, 40(10):1-9.

[28]. Bhat S P. Finite-time stability of continuous autonomous systems[J]. Siam J.contr.opti, 2000, 38(3):751-766.

[29]. Qian C, Lin W. A continuous feedback approach to global strong stabilization of nonlinear systems[J]. IEEE Transactions on Automatic Control, 2001, 46(7):1061-1079.

[30]. Babu K R, Sarma I G, Swamy K N. Switched bias proportional navigation for homing guidance against highly maneuvering targets[J]. Journal of Guidance Control and Dynamics, 1994, 17(6):13571363.

[31]. Shtessel Y B, Shkolnikov I A, Levant A. Guidance and Control of Missile Interceptor using SecondOrder Sliding Modes[J]. IEEE Transactions on Aerospace \& Electronic Systems, 2009, 45(1):110-124.

[32]. Levant. Higher-order sliding modes, differentiation and output-feedback control[J]. International Journal of Control, 2003, 76(9-10):924-941.

[33]. Huang X, Lin W, Yang B. Global finite-time stabilization of a class of uncertain nonlinear systems ઐ[J]. Automatica, 2005, 41(5):881-888. 
[34]. Phadke S B, Talole S E. Sliding Mode and Inertial Delay Control Based Missile Guidance[J]. IEEE Transactions on Aerospace \& Electronic Systems, 2012, 48(4):3331-3346.

[35]. He S, Wang W, Wang J. Discrete-Time Super-Twisting Guidance Law with Actuator Faults Consideration[J]. Asian Journal of Control, 2017, 19(5): 1854-1861.

[36]. Vaddi S, Menon P, Ohlmeyer E. Target state estimation for integrated guidance-control of missiles[C]//AIAA Guidance, Navigation and Control Conference and Exhibit. 2007: 6838. 
2018-09-21

pÿComposite finite time convergent guidance law for maneuvering targets pÿwith second order autopilot lag

\author{
Wu, Junxiong
}

Wiley

pÿWu J, Wang H, He S, Lin D. (2020) Composite finite time convergent guidance law for pÿmaneuvering targets with second order autopilot lag. Asian Journal of Control, Volume 22, Issue 1, January 2020, pp. 556-569 https://doi.org/10.1002/asjc. 1922

Downloaded from Cranfield Library Services E-Repository 